\title{
Poles and resonances in the coupled channels model for $\bar{K} N$ interaction
}

\section{Hrazdilová*}

Faculty of Nuclear Sciences and Physical Engineering, Czech Technical University in Prague,

Czech Republic

E-mail: hrazdlen@fjfi.cvut.cz

\section{A. Cieplý}

Nuclear Physics Institute, 25068 Rez, Czech Republic

E-mail: cieply@ujf.cas.cz

\begin{abstract}
We study dynamically generated resonances that arise as a result of low energy meson-baryon interaction in a chirally motivated coupled channel model. Our approach is based on a solution of the Lippman-Schwinger equation for the coupled meson-baryon channels $\pi \Lambda, \pi \Sigma, \bar{K} N, \eta \Lambda, \eta \Sigma$, and $K \Xi$ with an interaction kernel taken in a separable form. The resonances are searched for as poles of the T-matrix on unphysical Riemann sheets in the complex energy plane. We examine s-wave resonances with strangeness $S=-1$ with accent on isoscalar resonances $\Lambda(1405)$ and $\Lambda(1670)$. Additional insights are obtained by investigating movement of the poles to the zero coupling limit and to the $S U(3)$ symmetry restoraton limit.
\end{abstract}

50th International Winter Meeting on Nuclear Physics - Bormio2012,

23-27 January 2012

Bormio, Italy

* Speaker. 


\section{Introduction}

Baryon resonances play an important role in examining non-perturbative physics at low energies. In our work, we are interested in s-wave resonances with $S=-1$ strangeness and $I=0,1$ isospins that appear in kaon-nucleon interactions. The resonances are generated dynamically due to interaction of meson-baryon components and the T-matrix is obtained as a solution of coupled channel Lippman-Schwinger equation. In such approach, the $\Lambda(1405)$ resonance that governs the low energy $\bar{K} N$ physics is typically represented by two poles. The aim of the present work is to explore the pole content of our chirally motivated model up to energies around $1800 \mathrm{MeV}$ and get some insights on the origin of the poles. The latter is achieved by by following the pole movements on the complex energy manifold into the zero coupling limit and to the $S U(3)$ symmetry limit.

We employ chirally motivated meson-baryon potentials $V_{i j}$ taken in a separable form

$$
V_{i j}\left(k, k^{\prime}, \sqrt{s}\right)=\sqrt{\frac{1}{2 E_{i}} \frac{M_{i}}{\omega_{i}}} g_{i}(k) \frac{C_{i j}(\sqrt{s})}{f^{2}} g_{j}\left(k^{\prime}\right) \sqrt{\frac{1}{2 E_{j}} \frac{M_{j}}{\omega_{j}}} \quad, \quad g_{i}(k)=\frac{1}{1+\left(\frac{k}{\alpha_{i}}\right)^{2}},
$$

where $g_{i}$ are the off-shell form factors with $\alpha_{i}$ standing for the inverse range of the interaction. The parameter $f$ denotes the pseudoscalar meson decay constant and $M_{i}, E_{i}$ and $\omega_{i}$ represent the baryon mass, meson and baryon energies in the c.m. frame in the $i$-th channel. The underlying chiral $S U(3)$ symmetry is reflected by the structure of the $C_{i j}$ coefficients (specified in Refs. [1], [3]) that include terms up to the second order in the meson c.m. kinetic energies. The indeces $i$ and $j$ run over the set of coupled meson-baryon channels composed from the $\pi \Lambda, \pi \Sigma, \bar{K} N, \eta \Lambda, \eta \Sigma$, and $K \Xi$ states (and considered either as the relevant charged states, 10 channels in total, or states with the appropriate isospin, 4 channels for $I=0$ and 5 channels for $I=1$ ).

The resonances manifest themselves as poles of the transition matrix. The pole positions are identified as solutions $z_{R}$ of the equation that sets to zero the determinant of the inverse of the T-matrix,

$$
\operatorname{det}\left|T^{-1}(z)\right|=0
$$

where $z$ stands for a complex energy at a specific Riemann sheet. The masses and widths of the resonances assigned to the poles can then be approximated by the relation

$$
z_{R} \approx M_{R}-i\left(\Gamma_{R} / 2\right)
$$

\section{Model comparison}

In the present report, we show a pole analysis performed for three alternate models: TW1 [2], NLO30 [2] and CS30 [1]. The simplest TW1 model incorporates only the leading TomozawaWeinberg term of the chiral Lagrangian whereas the NLO30 and CS30 models include the leading (LO) plus the next-to-leading (NLO) orders of the underlying Lagrangian. The free parameters of the models were fitted to the available experimental data on the low energy $\bar{K} N$ interaction, namely: the $K^{-} p$ cross sections (see references collected in [3]), $K^{-} p$ threshold branching ratios [4] and the kaonic hydrogen characteristics reported by the DEAR [5] and SIDDHARTA [6] collaborations. While the fresh SIDDHARTA data were used for the TW1 and NLO30 models, the CS30 model 


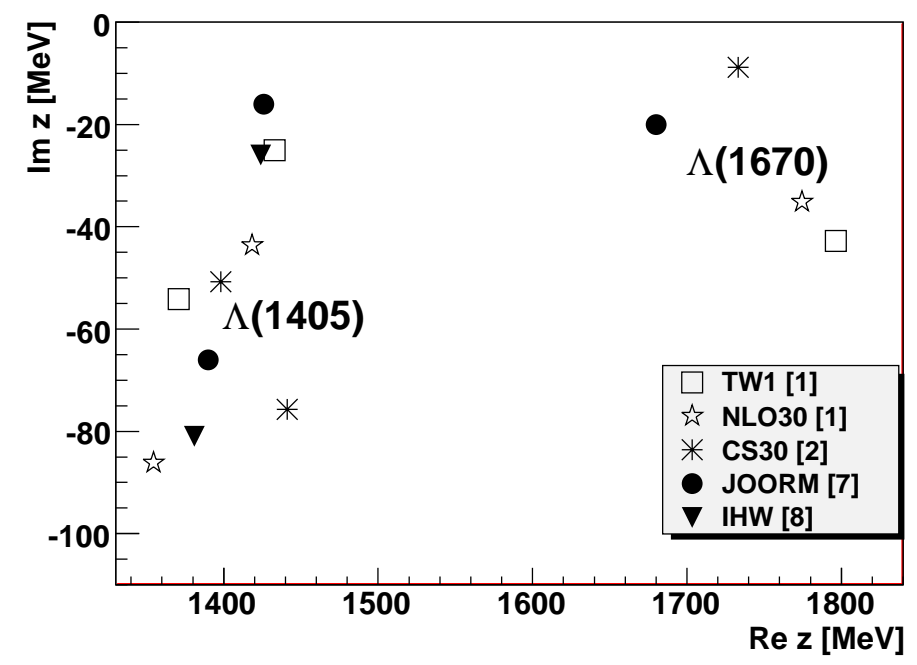

Figure 1: The positions of the isoscalar poles assigned to the $\Lambda(1405)$ and $\Lambda(1670)$ resonances in the TW1, NLO30 and CS30 models are shown in comparison with two other LO models JOORM [7] and IHW [8]. Each model generates two poles assigned to the $\Lambda(1405)$ resonance.

employed the older DEAR data. More details on the models and on the fitting procedure can be found in Refs. [1], [2].

In figure 1 we present in comparison the positions of the isoscalar poles as provided by various models. The $\Lambda(1405)$ resonance is represented by two poles $z_{1}$ and $z_{2}$ found on the second Riemann sheet $(\mathrm{RS})[-+++]$, where the signs are those of the imaginary parts of the c.m. momenta in the $\pi \Sigma, \bar{K} N, \eta \Lambda$ and $K \Xi$ channels, respectively. Surprisingly, the the experimental value of the $\Lambda(1405)$ mass and the resonance width are well reproduced by the $z_{2}$ position in the LO models and the NLO terms introduced in our NLO30 and CS30 models increase the imaginary part of the pole energy. The position of the $z_{1}$ pole at lower energies seems to be more model dependent. Though, due to a significantly larger imaginary part of the $z_{1}$ pole, it should not substantially affect the physical observables.

The pole assigned to the $\Lambda(1670)$ is located at rather varied positions for the considered models. All our models (TW1, NLO30 and CS30) generate the pole at energies about 50-100 MeV higher than the JOORM model that agrees much better with the experimental value of the $\Lambda(1670)$ mass. Nevertheless, it is important to emphasise that our models are fitted solely to the $\bar{K} N$ data at threshold and for very low kaon momenta. Thus we do not expect the models to work so well at higher energies.

\section{Zero coupling limit and SU(3) restoration limit}

The origin of the observed poles can be traced to their positions in a hypothetical situation when the interchannel couplings are switched off. In figure 2 we show the trajectories of the poles as they move on the complex energy manifold as the interchannel couplings are gradually reduced by a factor $x$, i.e. using $x \cdot C_{i j}$ for $i \neq j$ and going from physical couplings $(x=1)$ to the zero coupling limit $(x=0)$. For $x=0$ only the diagonal chiral couplings $C_{i j} \delta_{i j}$ remain nonzero and each 
pole may be assigned to a pertinent uncoupled channel in which it persist. It can be shown that only channels with nonzero diagonal couplings $C_{i i}$ can have a pole for $x=0$ and the pole evolves to its position in the physical limit $(x=1)$ due to interaction with other channels.
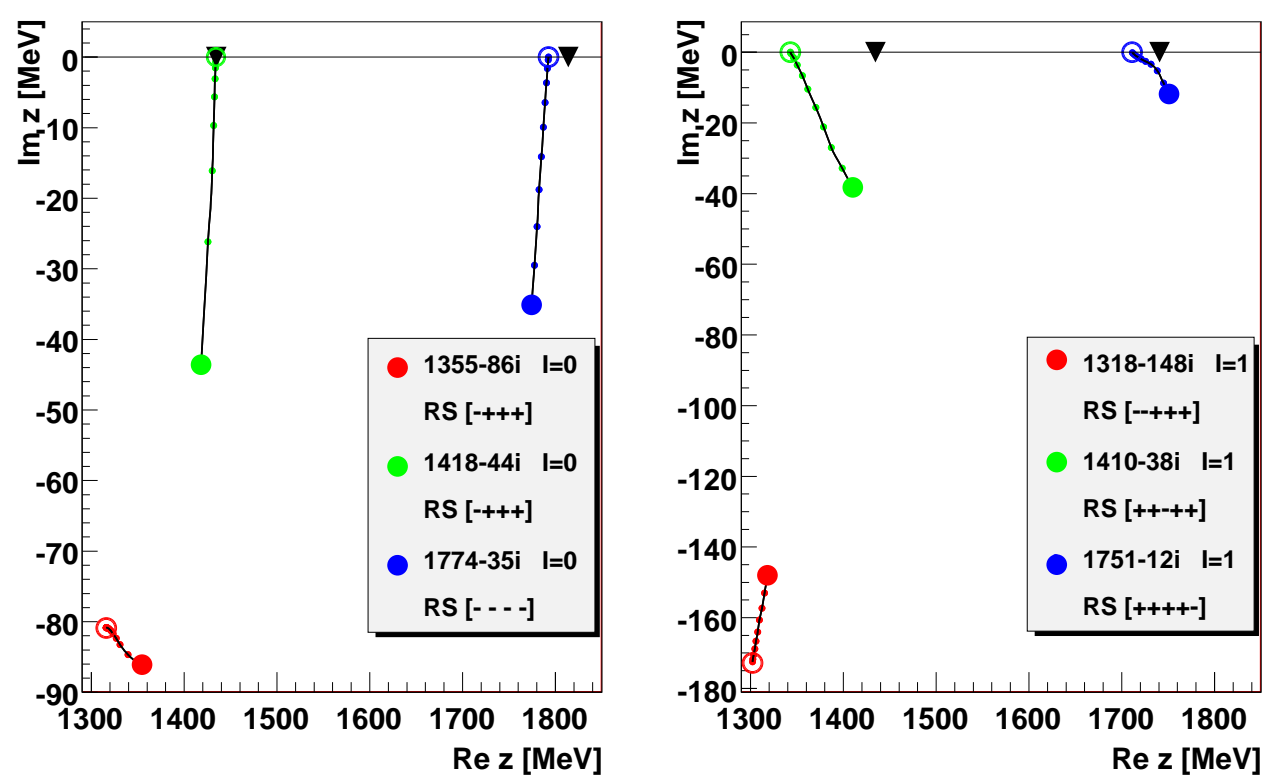

Figure 2: Pole movements upon scaling the nondiagonal interchannel couplings. Left panel: isoscalar states $(I=0)$, right panel: isovector states $(I=1)$. The large solid and empty circles show the pole positions in the physical and zero coupling limits, respectively. The triangles mark the $\bar{K} N$ and $K \Xi$ thresholds and the Riemann sheets the poles move on are specified in the legend.

The figure 2 visualizes the movement of the poles for the NLO30 model. The picture is very similar for the other models, though the exact pole positions do vary to some extend for $x>0$. In the left panel, the trajectories of poles assigned to $\Lambda(1405)$ and $\Lambda(1670)$ resonances are shown. In the zero couplig limit, the $\Lambda(1405)$ poles are represented by a resonance in the $\pi \Sigma$ channel and a bound state in the $\bar{K} N$ channel. The pole assigned to $\Lambda(1670)$ ends its movement for $x=0$ under the $K \Xi$ threshold on the unphysical Riemann sheet and can be identified with a $K \Xi$ quasi-bound state. Similar conclusions can be reached for the three $I=1$ poles shown in the right panel of figure 2.

Drawing an inspiration from Ref. [7], we also studied the movement of the poles when going to the limit of a restored $S U(3)$ symmetry. Following Ref. [7] we vary the hadron masses from their physical values to their $S U(3)$ chiral limits represented by $m_{0}=370 \mathrm{MeV}$ for the mesons and $M_{0}=1150 \mathrm{MeV}$ for the baryons. This is achieved by an introduction of a scaling factor $x_{S U 3}$ and by expressing the meson and baryon masses as

$$
M_{i}\left(x_{S U 3}\right)=M_{0}+x_{S U 3}\left(M_{i}-M_{0}\right) \quad, \quad m_{i}^{2}\left(x_{S U 3}\right)=m_{0}^{2}+x_{S U 3}\left(m_{i}^{2}-m_{0}^{2}\right) .
$$

Then, $x_{S U 3}=1$ represents the physical limit and $x_{S U 3}=0$ the limit of the restored $S U(3)$ symmetry. In figure 3 we show the pole trajectories for the TW1 model when varying the value of $x_{S U 3}$ from 1 to 0 . We confirm the results of Ref. [7] concerning the restoration of the $S U(3)$ singlet and octet 
states in the $x_{S U 3}=0$ limit. However, unlike the authors of Ref. [7] we observe an additional $I=1$ state (related to an isovector $\pi \Sigma$ resonance in the zero coupling limit) that goes to the $S U(3)$ octet for $x_{S U 3}=0$. We also note that the other $I=1$ state that evolves from the $S U(3)$ octet (and relates to $\bar{K} N$ channel in the zero coupling limit) does not get lost on its way to the physical limit while in Ref. [7] the state disappeared for $x_{S U 3} \approx 0.6$ due to a different treatment of the Riemann sheets.

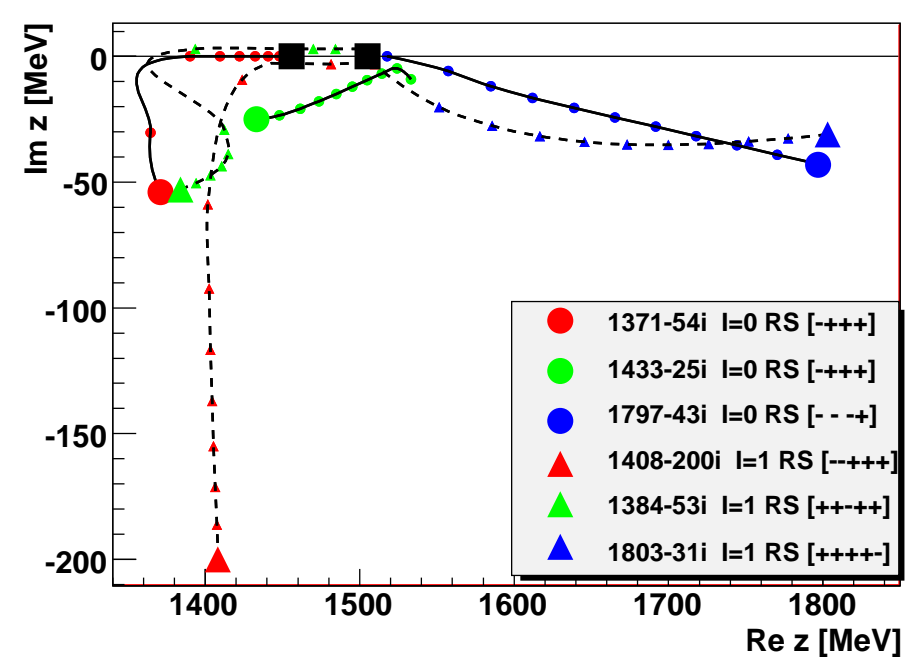

Figure 3: Trajectories of isoscalar (continuous lines) and isovector (dashed lines) poles obtained by varying the $S U(3)$ scaling parameter from $x_{S U(3)}=1$ to $x_{S U(3)}=0$. Full circles $(I=0)$ and triangles $(I=1)$ correspond to physical values of meson and baryon masses, the full squares represent the positions of the $S U(3)$ singlet and octet states in the $S U(3)$ restoration limit.

\section{References}

[1] A. Cieplý and J. Smejkal, Separable potential model for $K^{-} N$ interactions at low energies, Eur. Phys. J. A 43 (2010) 191 [arXiv:0910.1822 [nucl-th] ].

[2] A. Cieplý and J. Smejkal, Chirally motivated $\bar{K} N$ amplitudes for in-medium applications, (2012) [arXiv:1112.0917v2 [nucl-th]].

[3] N. Kaiser, P.B. Siegel and W. Weise, Chiral dynamics and the low-energy kaon - nucleon interaction, Nucl. Phys. A 594 (1995) 325 [arXiv: nucl-th/ 9505043 ].

[4] A.D. Martin, Kaon - Nucleon Parameters, Nucl. Phys. B 179 (1981) 33; and earlier references cited therein.

[5] G. Beer et al. [DEAR Collab.], Measurement of the kaonic hydrogen X-ray spectrum, Phys. Rev. Lett. 94 (2005) 212302 [arXiv:0910.1822 [nucl-th] ].

[6] M. Bazzi and others, A New Measurement of Kaonic Hydrogen X rays, Phys. Lett. $\mathbf{B 0 4}$ (2011) 113 [arXiv:1105.3090 [nucl-ex]].

[7] D. Jido, J.A. Oller, E. Oset, A. Ramos and U.-G. Meissner, Chiral dynamics of the two Lambda(1405) states, Nucl. Phys. A 725 (2003) 181 [arXiv:nucl-th/0303062].

[8] Y. Ikeda, T. Hyodo and W. Weise, Improved constraints on chiral SU(3) dynamics from kaonic hydrogen, Phys. Lett. B 706 (2011) 63 [arXiv:1109.3005 [nucl-th]]. 